\title{
Síntomas neuropsiquiátricos en encefalitis por anticuerpos contra el receptor N-metil-D-aspartato: a propósito de dos casos
}

\author{
Neurophychiatric symptoms in anti-N-methyl-D-aspartate receptor \\ encephalitis: apropos of two cases
}

\author{
N Custodio ${ }^{1,2, a, b}$, J Escobar-Montalvo 1,2,c \\ ${ }^{1}$ Unidad de Investigación del Instituto Peruano de Neurociencias. \\ ${ }^{2}$ Unidad de Diagnóstico de Deterioro Cognitivo y Prevención de Demencias de la Clínica Internacional. \\ ${ }^{a}$ Magister en Medicina, Universidad de San Martin de Porres; ${ }^{b}$ Master en Neurociencias, Universidad Europea Miguel de Cervantes; \\ ${ }^{\circ}$ Máster de Investigación en Inmunología, Universidad Complutense de Madrid.
}

\begin{abstract}
Resumen
La encefalitis frente al receptor de N-metil-D-aspartato (NMDAR) está incluida en el grupo de las encefalitis autoinmunes. Es más frecuente en mujeres en edad reproductiva. Las manifestaciones clínicas iniciales pueden ser similares a los pródromos de las infecciones virales. En ocasiones se asocia con la presencia de neoplasias. El diagnóstico oportuno y la instauración precoz del tratamiento inmunomodulador es determinante en el pronóstico. En el presente artículo, se realiza una revisión de la literatura y se presentan dos casos de encefaltis anti-NMDAR en diferentes edades de la vida.

Palabras clave. Receptores, N-metil-D-aspartato; Anticuerpos; Encefalitis.

Abstract

Encephalitis against N-methyl-D-aspartate receptor (NMDAR) is included in the group of autoimmune encephalitis. It is more common in women in reproductive age. The first clinical features may be similar to viral infections prodromes. It is sometimes related to neoplasms. The accurate diagnosis and early administration of immunomodulatory therapy is critical to the prognosis. In this article, a review of the literature was performed and two cases of anti-NMDAR encephalitis in different stages of life are presented.
\end{abstract}

Keywords. Receptors, N-methyl-D-aspartate; Antibodies; Encephalitis.

An Fac med. 2016;77(3):277-81 / http://dx.doi.org/10.15381/anales.v77i3.12419

\section{INTRODUCCIÓN}

Las encefalitis son procesos inflamatorios que afectan al sistema nervioso central, de etiología infecciosa, tumoral o autoinmune. En el contexto de las encefalitis autoinmunes, Dalmau y colaboradores describieron la existencia de un tipo de encefalitis relacionada con una respuesta antígeno anticuerpo frente a la porción externa del receptor del N-metil-D-aspartato (NMDAR), ubicados principalmente en neuronas hipocampales ${ }^{(1)}$. A la fecha se han descrito más de 500 casos a nivel mundial ${ }^{(1)}$. Sin embargo; en el momen- to actual no se han estimado tasas de prevalencia. Del total de los pacientes diagnosticados se ha observado que a pesar de detectarse anticuerpos antiNMDAR en varones, la mayoría de casos (alrededor del 80\%) se presenta en mujeres, como ocurre en otras enfermedades autoinmunes. La edad media de presentación es de 21 años; sin embargo, se han descrito casos pacientes con edades entre los 8 meses hasta 85 años de edad (2). Entre septiembre 2007 y febrero 2011, en California se desarrolló un estudio epidemiológico en 761 pacientes con encefalitis $(\mathrm{C} a$ liformia Encephalitis Project), en el cual se encontró que la encefalitis antiNMDAR fue la causa más frecuente de encefalitis de etiología indeterminada, siendo 4 veces más frecuente que la infección por: enterovirus, VHS-1, virus del Nilo Occidental o virus Varicela Zoster. El $65 \%$ de la encefalitis antiNMDAR ocurrió en pacientes $\leq 18$ años. Se observó una predilección por el sexo femenino en las encefalitis antiNMDAR, no observada en las encefalitis virales. Además, las convulsiones, la disfunción del lenguaje, la psicosis y las alteraciones electroencefalográficas fueron significativamente más frecuentes en pacientes con encefalitis anti- 
NMDAR (la inestabilidad autonómica se produjo exclusivamente en este grupo) ${ }^{(3)}$. En otro estudio epidemiológico de encefalitis realizado en Inglaterra en 203 pacientes, se observó que 4,4\% de pacientes con encefalitis de etiología indeterminada eran causadas por encefalitis anti-NMDAR. Asimismo, se observó que la infección por VHS-1 y la encefalomielitis diseminada aguda fueron las 2 entidades más comunes, seguidas por la encefalitis anti-NMDAR ${ }^{(4)}$.

Cabe destacar que en los primeros casos se consideró a la encefalitis anti-NMDAR como un síndrome paraneoplásico por su coexistencia con tumores ováricos (principalmente teratomas). Sin embargo, en la actualidad se observa que la frecuencia de teratomas en mayores de 18 años es de $56 \%$ y en menores de 18 años de aproximadamente $30 \%$. Asimismo, es importante comentar que la presentación de tumores en niños es prácticamente inexistente. Cabe destacar que el teratoma es la neoplasia más frecuente que se asocia a la encefalitis anti-NMDAR. No obstante se han descrito otras neoplasias, como teratomas mediastinales, tumores estromales, neuroblastomas y linfoma de Hodgkin ${ }^{(5)}$.

Los NMDARs son canales iónicos permeables al calcio que presentan una estructura convencional formada, principalmente, por complejos tetraméricos compuestos de la subunidad NR1 unida a glicina y las subunidades NR2 (NR2A-NR2D) unidas a glutamato. Estas subunidades se ensamblan entre sí para formar otros subtipos de receptores con distintas localizaciones sinápticas, propiedades fisiológicas y farmacológicas, y propiedades de señalización intracelular. Fisiológicamente, los NMDAR están vinculados con el desarrollo del sistema nervioso, la generación de ritmos respiratorios, la locomoción y procesos de aprendizaje, memoria y neuroplasticidad ${ }^{(2)}$. En la actualidad se postula que la hiperactividad de los NMDARs induce muerte neuronal ocasionada por excitotoxicidad post sináptica y una regulación positiva persistente de la función de receptores AMPA, que conducirían a la potenciación a largo plazo de las alteraciones hipocampales. Esta hiperactividad es un mecanismo propuesto para explicar las crisis epilépticas y el deterioro cognitivo. Mientras tanto, la disminución de la actividad de los NMDAR se asocia con síntomas psicóticos ${ }^{(2)}$. Presentamos la presente comunicación con la finalidad de analizar los particulares síntomas neuropsiquiátricos, la fisiopatología y el tratamiento de dos pacientes con probable encefalitis anti-NMDAR.

\section{CASOS CLÍNICOS}

\section{Caso 1:}

Paciente de sexo femenino de 4 años de edad, sin antecedentes de riesgo perinatal, presentó un tiempo de enfermedad de 5 semanas, con inicio de 3 días de tos, elevación de temperatura no medida y malestar general. Una semana después, tuvo episodios diarios y repetidos de automatismos de menos de un minuto de duración (mirada fija y fenómenos de masticación en número de 4 a 5 por día) con recuperación completa después de los episodios, por lo que recibió tratamiento con carbamazepina a dosis de $10 \mathrm{mg} / \mathrm{kg} / \mathrm{día}$, sin alivio de los episodios. Luego de 2 semanas, presentó dos episodios similares, seguidos de crisis generalizada tónico-clónica, por lo que se indicó incrementar las dosis a $15 \mathrm{mg} / \mathrm{kg} / \mathrm{día}$. Luego de estos episodios, mostró irritabilidad, labilidad emocional, agitación psíquica de predominio nocturno y dificultades en el lenguaje, caracterizado por preservación de la comprensión, con alteraciones de la expresión asociado a anomia y anartria. El día anterior al ingreso tuvo 3 crisis tónico-clónicas generalizadas, con agitación psíquica nocturna y mioclonías de hemicara derecha. En el primer día de hospitalización, sus funciones vitales, examen clínico y neurológico revelaron afasia de Broca e hiperreflexia asimétrica. El recuento de leucocitos y la proteína $\mathrm{C}$ reactiva fueron ligeramente elevados. El análisis citoquímico del líquido cefalorraquídeo (LCR) fue normal. Se decidió iniciar tratamiento con dexametasona y aciclovir por el supuesto de encefalitis viral, y valproato de sodio a dosis de $40 \mathrm{mg} / \mathrm{kg} / \mathrm{día}$. Los cultivos bacterianos en sangre y de LCR fueron negativos. Los cultivos virales y el análisis de reacción en cadena de polimerasa para virus de herpes simple y Mycobacterium tuberculosis también fueron negativos, por lo que se detuvo el tratamiento con aciclovir. La tomografía espiral multicorte (TEM) cerebral con contraste y la imagen por resonancia magnética (IRM) cerebral con gadolinio no demostraron cambios. El electroencefalograma (EEG) estándar reveló enlentecimiento difuso de las ondas, pero no descargas epilépticas. A la semana del ingreso, debido a persistencia de crisis parciales complejas y mioclonías de hemicara derecha se decidió agregar lamotrigina a dosis de titulación de $0,2 \mathrm{mg} / \mathrm{kg} / \mathrm{día}$ hasta una dosis de mantenimiento de $2 \mathrm{mg} / \mathrm{kg} / \mathrm{día}$ y realizar una segunda punción lumbar para estudio de proteína 14-3-3 y anticuerpos anti-NMDAR. Una semana después de obtener la muestra de LCR se informó anticuerpos positivos antiNMDAR, por lo que diagnosticamos encefalitis anti-NMDAR. Se realizó imágenes para ecografía de tiroides, tomografía de tórax, mediastino y abdominopélvica, siendo informadas como normales. Se discutió la posibilidad de iniciar tratamiento con inmunoglobulina endovenosa, pero dada la buena evolución (control de crisis parciales y generalizadas, agitación psíquica y lenguaje expresivo) con los anticonvulsivantes y corticoides, se optó por continuar el tratamiento con deflazacort en gotas a dosis de $1 \mathrm{mg} / \mathrm{kg} / \mathrm{día}$, el cual se mantuvo por 6 meses. Un año después, ella continúa con valproato de sodio, no presenta crisis epilépticas, ha recuperado las funciones del lenguaje y acude regularmente al colegio de educación inicial.

\section{Caso 2:}

Paciente de sexo masculino de 31 años de edad, de profesión ingeniero civil, con antecedentes de convulsión febril 
simple a los 2 años de edad y problemas de aprendizaje en la educación primaria asociado a déficit de atención sin hiperactividad, diagnosticados a los 8 años de edad y tratados con estrategias cognitivo-conductuales hasta los 12 años de edad. Un año antes de su ingreso, presentó un cuadro clínico caracterizado por disminución de la atención, pérdida del interés por actividades laborales y de entretenimiento, anhedonia y humor deprimido, siendo tratado con escitalopram, con buena respuesta, por lo que el paciente suspendió el tratamiento al tercer mes. Cuatro meses más tarde, presentó un primer episodio maniaco, caracterizado por logorrea, conducta desinhibida y agresiva, que remitió totalmente con dosis de quetiapina $200 \mathrm{mg}$ tid, y se planteó el diagnóstico de desorden bipolar. Dos meses más tarde mostró un severo cuadro depresivo y se inició tratamiento con aripiprazole, litio, valproato y escitalopram, sin remisión de síntomas. Un mes antes del ingreso tuvo un episodio de automatismo de marcha militar de 30 a 40 segundos de duración, seguido de confusión, desorientación y dolor de cabeza por 3 a 4 horas, que se repitió en tres oportunidades en el curso de tres semanas. El día anterior al ingreso, sufrió un episodio similar, seguido de una crisis generalizada tónico-clónica. En el examen clínico de ingreso, los signos vitales fueron normales y el examen neurológico reveló disminución del balanceo de miembro superior izquierdo, pasos cortos y asimétricos, con discreta triple flexión en el examen de marcha, no evidencia de temblor de actitud y postural, pero sí una marcada rigidez extrapiramidal en hemicuerpo izquierdo. Los reflejos osteotendinosos fueron normales y los reflejos patológicos ausentes. Se decidió retirar la medicación e iniciar oxcarbazepina a $300 \mathrm{mg}$ bid, titulando hasta $600 \mathrm{mg}$ bid, hidratación parenteral y midazolan condicional a agitación psíquica y/o crisis convulsivas. El examen de nervios craneanos fue normal. La primera punción lumbar, imágenes cerebrales por TEM, IRM y EEG estándar fueron normales. Se realizó estudios para enfermedades reumatológicas (artritis reumatoide, lupus y otras), metabólicas (diabetes, hipotiroidismo), anti-fosfolipídicos (anticoagulante lúpico y anticardiolipina), deficiencia de vitaminas (vitamina $A$, B1, B6 y B12), infecciosas (VIH, sífilis, tuberculosis) y marcadores paraneoplásicos (anticuerpos neuronales nucleares 1 y 2 - anti $\mathrm{Hu}$, y anticuerpos citoplásmicos de células de Purkinge-anti Yo), siendo informados como normales. Las imágenes para ecografía de tiroides, tomografía de tórax, mediastino y abdominopélvica fueron normales. El paciente persistió con crisis de agitación psíquica y crisis parciales complejas, por lo que se agregó risperidona $2 \mathrm{mg}$ tid y levetiracetam $1000 \mathrm{mg}$ bid y se programó otra IRM y una segunda punción lumbar para estudio de anticuerpos anti-NMDAR, reacción en cadena de polimerasa para virus de herpes simple y Mycobacterium tuberculosis, y proteína 14-3-3. La IRM no mostró cambios. A la espera de los resultados y con el citoquímico normal, se inició tratamiento con pulso de metilprednisolona a dosis de $1 \mathrm{mg} / \mathrm{kg} /$ día por 5 días. Al segundo día de tratamiento, la agitación psíquica fue controlada, pero persistían las crisis parciales complejas (aunque disminuyeron de 4 a 6 hasta 2 a 3 en 24 horas). Al octavo día de iniciado el primer pulso de metilprenisolona, se obtuvo el reporte de anticuerpos positivos anti-NMDAR, por lo que se decidió un segundo pulso de metilprednisolona e infusión de inmunoglobulinas en dosis de $2 \mathrm{~g} / \mathrm{kg}$ de peso repartida en 5 sesiones en días consecutivos. Luego del tercer día de tratamiento con inmunoglobulinas, las crisis parciales complejas fueron controladas totalmente. Luego de permanecer hospitalizado 20 días, el paciente fue dado de alta sin crisis de agitación psíquica, sin crisis epilépticas, con hipertonía extrapiramidal en hemicuerpo izquierdo, con programa para evaluación neuropsicológica, con levodopa-benserazida 200/50 mg media tableta 7 am$12 \mathrm{~m}-5 \mathrm{pm}$, oxcarbazepina $600 \mathrm{mg}$ bid y levetiracetam $1000 \mathrm{mg}$ bid. Al mes del alta, la evaluación neuropsicológica reveló trastorno de las funciones ejecutivas y de la atención y el tono muscular fue normal, por lo que se decidió retirar el tratamiento con levodopa/carbidopa. Un año después, la evaluación neuropsicológica fue normal, continuaba en tratamiento con levetiracetam 1000 mg bid, y se reintegró paulatinamente a sus actividades habituales.

\section{DISCUSIÓN}

A pesar que los primeros casos de encefalitis anti-NMDAR fueron reportados en mujeres jóvenes en edad reproductiva, esta patología también se presenta en poblaciones de edad pediátrica y adulta, grupos de edad en los cuales no se ha observado una clara tendencia dependiente del género; mientras tanto, en la población en edad reproductiva se observa una elevada frecuencia en sexo femenino (proporción en género femenino/masculino: 4/1) ${ }^{(4)}$. En la edad adulta, un estudio sugiere que el inicio de los primeros síntomas puede variar entre ambos géneros. En pacientes de sexo masculino, los síntomas iniciales suelen ser neurológicos, principalmente crisis parciales (comparado con crisis generalizadas del sexo femenino), mientras que en pacientes de sexo femenino, los síntomas iniciales suelen ser síntomas psiquiátricos ${ }^{(6)}$. En el caso 2, los síntomas iniciales fueron psiquiátricos, desarrollando luego síntomas neurológicos. De acuerdo con lo reportado, el desarrollo y progresión de los síntomas tiene estadios clínicos bien definidos. Como sucedió en el caso 1, la mayoría de los pacientes (70\%) desarrolla una fase prodrómica caracterizada por febrícula, malestar general, náuseas y dolor de cabeza que puede durar 5 días a 2 semanas, después del cual progresa hacia una fase psicótica, con cambios emocionales y conductuales, deterioro cognitivo, miedo, paranoia, hasta llegar a delusiones y alucinaciones. Luego se presentan los síntomas de fase neurológica, con crisis epilépticas y en algunos casos pueden llegar a una fase catatónica, mostrando signos de disfunción autonómica ${ }^{(7)}$. Los síntomas de compromiso de lenguaje son frecuentes y de instalación lenta y progresiva, como sucedió en el 
caso 1. Sin embargo, el reporte de súbita y aislada afasia de Broca es infrecuente ${ }^{(8)}$. En el diagnóstico diferencial debe considerarse la afasia secundaria a crisis convulsivas, similar a la parálisis de Tod y síndrome de Landau Kleffner ${ }^{(7)}$. En el caso 2, los síntomas de depresión y psicosis fueron de instalación lenta y progresiva en intensidad, hasta progresar a síntomas neurológicos, evidenciado por crisis parciales complejas secundariamente generalizadas y extrapiramidalismo unilateral. Debido a que los síntomas psiquiátricos se presentan en etapas tempranas de la enfermedad, aproximadamente el $70 \%$ de los pacientes son evaluados inicialmente por un psiquiatra. Peor aún, existen escasos casos de encefalitis anti-NMDAR donde los síntomas psiquiátricos se presentan por largos periodos antes de la aparición de síntomas neurológicos, como el caso reportado por Simabukuro y col. (9) de un paciente con larga historia de desorden bipolar que presentó luego de 10 años un episodio psicótico seguido de discinesias orofaciales asociadas a alza térmica. Y los casos, donde los síntomas psiquiátricos son la única expresión, recientemente llaman la atención, pues existe una clara relación entre esquizofrenia y trastornos de la autoinmunidad. Se ha demostrado un síndrome clínico de encefalitis anti-NMDAR con síntomas psicóticos relacionados a anticuerpos GluN1. No obstante, aún no se establece su independencia de síntomas neurológicos y la posibilidad de estrategias terapéuticas ${ }^{(10)}$.

En relación a los mecanismos inmunológicos relacionados a los casos de encefalitis anti-NMDAR, se ha observado un fenómeno de autoinmunización debido a una sobreproducción de autoanticuerpos frente al NMDAR que ocasiona una reacción cruzada que conduce a la internalización celular del receptor mediante autofagia ${ }^{(1,11)}$. Los mecanismos propuestos de la inducción de autoinmunización en la encefalitis anti-NMDAR son los siguientes (ver figura 1):

- Transformación de linfocitos T y B: los tumores (teratomas) originan autoantígenos desconocidos que entran en contacto con las células presentadoras de antígenos ${ }^{(11)}$. Estas últimas presentan los autoantígenos a los linfocitos $\mathrm{T}$, lo que ocasiona un incremento de las concentraciones de citoquinas, que atravesarán la barrera hematoencefálica (BHE) y la estimulación de los linfocitos $B$ que se transformarán en células plasmáticas para la formación de anticuerpos específicos contra los autoantígenos tumorales ${ }^{(12,13)}$. Esto provoca un fenómeno de reactividad cruzada que modula las subunidades del NMDAR NR2A y NR2B del neocórtex cerebral y del hipocampo, induciendo un incremento del paso de calcio al medio intracelular, lo que ocasiona muerte neuronal por excitotoxicidad ${ }^{(11,14)}$.

- Autoinmunidad anti-NMDAR posiblemente inducida por patógenos: en los casos de encefalitis anti-NMDAR no asociados a tumores, se sospecha que la pérdida de tolerancia y la inducción de autoinmunidad podría estar activada por una infección viral. Esto se cree porque la mayoría de los pacientes presentan pródromos similares a los que se preceden las infecciones virales. Esta hipótesis postula a los retrovirus endógenos como patógenos que podrían inducir autorreactividad en los casos de encefalitis anti-NMDA ${ }^{(14,15)}$.

- El sistema inmune innato regula la autoinmunidad adaptativa: los tumores son fuentes de ligandos para los receptores de reconocimiento de patrones (RPR) como TLR, NOD-R, RIG-1 y MDA-5. Esta sobreproducción de ligandos ocasiona un incremento de la formación de HLA-I y HLA-II, que causa una alteración en la expresión de las moléculas CD80 y CD86 en las células presentadoras de antígenos, provocando una sobreproducción de IL-2 e IL6 que condiciona una maduración excesiva de LT, lo que podría ocasionar una pérdida de los mecanismos de tolerancia inmunológica, induciendo la generación de autoinmunidad ${ }^{(14,16,17)}$.
- Disrupción de la barrera hematoencefálica: la sobrestimulación del sistema inmune innato (sobreproducción de citoquinas y ligandos) incrementa la permeabilidad de la barrera hematoencefálica a especies reactivas de linfocitos $\mathrm{B}$ en el SNC. Asimismo, un incremento de los niveles de TGF- $\beta$ y de IL- 6 que actúan como factores estimulantes de los linfocitos $\mathrm{T}$ nativos para la formación de linfocitos Th17 que generan la producción de IL-22, IL-23 e IL17. Esta última interleucina ocasiona daño de las uniones delgadas (tight junctions) de las células endoteliales de la BHE, contribuyendo al incremento de la permeabilidad ${ }^{(14,17)}$.

- Autoinmunidad inducida por NMDARs formados fuera del sistema nervioso: los NMDARs pueden ser formados en localizaciones distintas al SNC (riñón, pulmón, miocardio, linfocitos, megacariocitos, células beta del páncreas y paratiroides). Esta formación exógena de NMDARs podría generar respuestas autoinmunes que por reactividad cruzada podrían condicionar episodios de encefalitis ${ }^{(18,19)}$.

En relación al tratamiento, se ha observado que el inicio precoz con fármacos inmunomoduladores influye en el pronóstico clínico de manera favorable. En este sentido se han planteado las siguientes líneas de tratamiento:

- Primera línea: inmunoglobulina IV (0,4 g/kg/día, durante 5 días), metilprednisolona IV $(1 \mathrm{~g} / \mathrm{kg} /$ día, durante 5 días) y plasmaféresis ${ }^{(10,20,21)}$.

- Segunda línea: rituximab (375 mg/ $\mathrm{m} 2$ /semana, durante 4 semanas) en combinación con ciclofosfamida $(750 \mathrm{mg} / \mathrm{m} 2$, administrado conjuntamente con la primera dosis de rituximab y después se administran ciclos mensuales). La segunda línea de tratamiento es administrada si no se observa respuesta, tras 10 días de la aplicación de los fármacos de primera línea ${ }^{(10,20,21)}$.

- Debido a que entre el 20 a 25\% de los pacientes presentan recaídas, se 


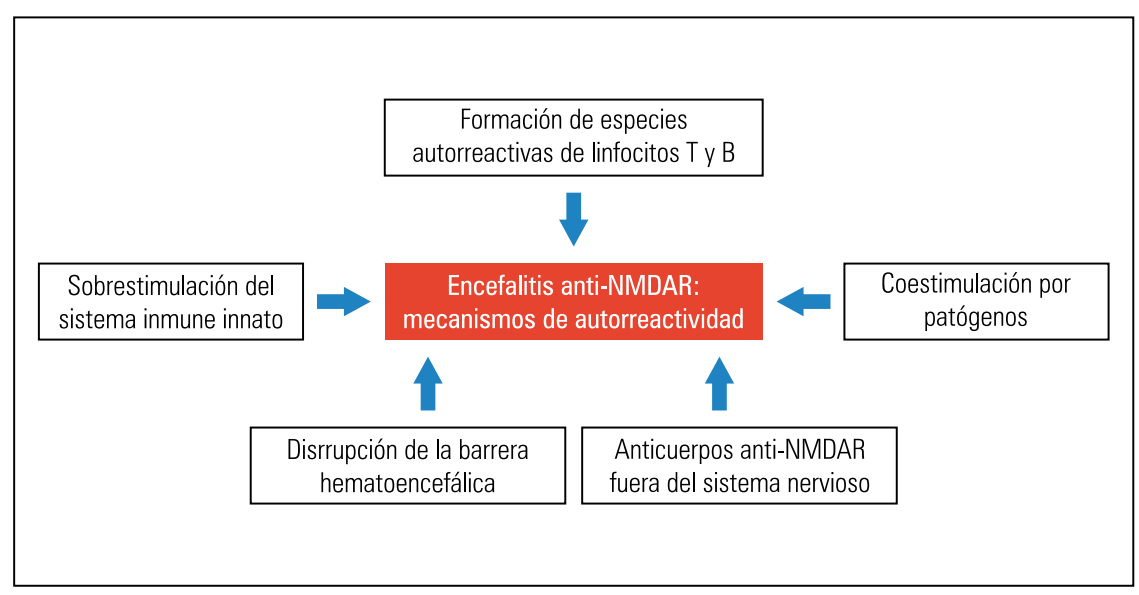

Figura 1. Mecanismos de autorreactividad: encefalitis anti-NMDAR.

recomienda continuar con el tratamiento inmunosupresor, tras la inmunoterapia inicial, con micofenolatomofetilo o azatioprina, durante al menos 1 año ${ }^{(10,20)}$.

- Otros tratamientos fármacos de mantenimiento, de control de crisis y de soporte vital del paciente. Además, es importante mencionar que algunas pacientes con teratomas ováricos presentan mejoría clínica tras el tratamiento quirúrgico ${ }^{(20,21)}$.

Con este esquema terapéutico se ha observado que aproximadamente el $75 \%$ de los casos muestra una recuperación completa o tiene secuelas leves, el 20\% secuelas moderadas o graves y aproximadamente un $5 \%$ mueren ${ }^{(10,20)}$.

En conclusión, dado que los síntomas neuropsiquiátricos son de presentación precoz y que el diagnóstico oportuno y la instauración precoz del tratamiento mejoran el pronóstico en los casos de encefalitis anti-NMDAR, es pertinente tener en cuenta esta patología en la práctica clínica diaria, ante un paciente joven que presenta síntomas psiquiátricos atípicos de inicio agudo y que evolucionan hacia síntomas neurológicos.

\section{REFERENCIAS BIBLIOGRÁFICAS}

1. Sansing L, Tüzün E, Ko M, Baccon J, Lynch D, Dalmau J. A patient with encephalitis associated with NMDA receptor antibodies. Nat Clin Pract
Neurol. 2007;3(5):291-6. http://dx.doi.org/10.1038/ ncpneuro0493.

2. Mann A, Grebenciucova E, Lukas R. Anti-N-methyld-aspartate-receptor encephalitis: diagnosis, optimal management, and challenges. Therapeutics and Clinical Risk Management. 2014;10:517-25. http://dx.doi.org/10.2147/TCRM.S61967.

3. Gable M, Sheriff H, Dalmau J, Tilley D, Glaser C. The frequency of autoimmune $\mathrm{N}$-methyl-Daspartate receptor encephalitis surpasses that of individual viral etiologies in young individual senrolled in the California Encephalitis Project. Clin Infect Dis. 2012 Apr;54(7):899-904. http://dx.doi. org/10.1093/cid/cir1038.

4. Granerod J, Ambrose H, Davies N, Clewley J, Walsh A, Morgan D, et al. Causes of encephalitis and differences in their clinical presentations in England: a multicentre, population-based prospective study. Lancet Infectious Diseases. 2010;10(12):835-44. http://dx.doi.org/10.1016/ S1473-3099(10)70222-X.

5. Ferdinand P, Mitchell L. Anti-NMDA receptor encephalitis. J Clin Cell Immunol. 2012;S10:1-6. http:// dx.doi.org/10.4172/2155-9899.S10-007.

6. Viaccoz A, Desestret V, Ducray F, Picard G, Cavillon $G$, Rogemond $V$, et al. Clinical specificities of adult male patients with NMDA receptor antibodies encephalitis. Neurology. 2014;82:556-63. http:// dx.doi.org/10.1212/WNL.0000000000000126.

7. Lazar-Molnar E, Tebo AE. Autoimmune NMDA receptor encephalitis. Clin Chim Acta. 2015;438:90-7. http://dx.doi.org/10.1016/j.cca.2014.08.010.

8. Deiva K, Pera MC, Maurey H, Chretien P, Archambaud $F$, Bouilleret $V$, et al. Sudden and isolated Broca's aphasia: a new clinical phenotype of anti NMDA receptor antibodies encephalitis in children. Eur J Paediatric Neurol. 2014;18:790-2. http:// dx.doi.org/10.1016/j.ejpn.2014.06.002.

9. Simabukuro MM, Freitas $\mathrm{CH}$, Castro LH. A patient with a long history of relapsing psychosis and mania presenting with anti-NMDA receptor encephalitis ten years after first episody. Dement Neurosychol. 2015;9(3):311-4. http://dx.doi. org/10.1590/1980-57642015DN93000016.

10. Titulaer M, McCracken L, Gabilondo I, Armangué T, Glaser C, lizuka T, et al. Treatment and prognostic factors for long-term outcome in patients with anti-NMDA receptor encephalitis: an observational cohort study. Lancet Neurol. 2013;12:157-65.
http://dx.doi.org/10.1016/S1474-4422(12)70310-1. 11. Dalmau J, Lancaster E, Martinez-Hernandez E, Rosenfeld M, Balice-Gordon R. Clinical experience and laboratory investigations in patients with anti-NMDAR encephalitis. Lancet Neurol. 2011 Jan;10(1):63-74. http://dx.doi.org/10.1016/S14744422(10)70253-2.

12. Tabata E, Masuda M, Eriguchi M, Yokoyama M, Takahashi Y, Tanaka K, et al. Immunopathological significance of ovarian teratoma in patients with anti-N-methyl-d-aspartate receptor encephalitis. Eur Neurol. 2014;71(1-2):42-8. http://dx.doi. org/10.1159/000353982.

13. Camdessanché J-P, Streichenberger N, Cavillon G, Rogemond V, Jousserand G, Honnorat J, et al. Brain immunohistopathological study in a patient with anti-NMDAR encephalitis. Eur J Neurol. 2011;18(6):929-31. http://dx.doi.org/10.1111/ j.1468-1331.2010.03180.x.

14. Ding H, Jian Z, Stary C, Yi W, Xiong X. Molecular pathogenesis of anti-NMDAR encephalitis. BioMed Research International. 2015;Article ID 643409:1-6. http://dx.doi.org/10.1155/2015/643409.

15. Prüss H, Finke C, Höltje M, Hofmann J, Klingbeil C, Probst $\mathrm{C}$, et al. N-methyl-D-aspartate receptor antibodies in herpes simplex encephalitis. Ann Neurol. 2012;72(6):902-11. http://dx.doi. org/10.1002/ana.23689.

16. Kawai T, Akira S. The role of pattern-recognition receptors in innate immunity: update on Toll-like receptors. Nat Immunol. 2010;11(5):373-84. http:// dx.doi.org/10.1038/ni.1863.

17. Rivest $\mathrm{S}$. Regulation of innate immune responses in the brain. Nat Rev Immunol. 2009;9(6):429-39. http://dx.doi.org/10.1038/nri2565.

18. Gill SS, Pulido OM. Glutamate receptors in peripheral tissues: current knowledge, future research, and implications for toxicology. Toxicol Pathol. 2001;29(2):208-23. http://dx.doi.org/10.1080/019 262301317052486.

19. Dickman KG, Youssef JG, Mathew SM, Said SI. Ionotropic glutamate receptors in lungs and airways: molecular basis for glutamate toxicity. Am J Respir Cell Mol Biol. 2004;30(2):139-44. http://dx.doi. org/10.1165/rcmb.2003-01770C.

20. Kruse JL, Jeffrey JK, Davis MC, Dearlove J, IsHak WW, Brooks JO. Anti-N-methyl-D-aspartate receptor encephalitis: a targeted review of clinical presentation, diagnosis, and approaches to psychopharmacologic management. Ann Clin Psychiatry. 2014;26(2):111-9.

21. Mann A, Machado NM, Liu N, Mazin A-H, Silver K, Afzal KI. A multidisciplinary approach to the treatment of anti-NMDA-receptor antibody encephalitis: a case and review of the literature. J Neuropsychiatry Clin Neurosci. 2012 May;24(2):247-54. http://dx.doi.org/10.1176/appi. neuropsych.11070151.

Artículo recibido el 16 de marzo de 2016 y aceptado para publicación el 9 de junio de 2016.

Conflictos de intereses: Ninguno.

Correspondencia:

Nilton Custodio.

Unidad de Investigación, Instituto Peruano de Neurociencias.

Dirección: Bartolomé Herrera 161, Lince.

Teléfono: 51-1-2653834.

Correo electrónico: ncustodio@ipn.pe 